\title{
Scanned Probe Microscopy: A Brief Tutorial
}

\author{
P. E. Russell
}

Materials Science and Engineering, and Analytical Instrumentation Facility

Campus Box 7531, North Carolina State University Raleigh, NC 27695-7531

This tutorial will introduce the basic concepts and modes of Scanned Probe Microscopy (SPM) including scanning tunneling microscopy (STM), atomic force microscopy (AFM). The most commonly utilized SPM techniques today are based on the basic AFM concept and include both contact mode (the original and most simple mode of AFM) variations and a wide range of oscillating tip modes sometimes referred to as AC modes, non-contact, intermittent contact or tapping modes. The basic instrumentation required of all modes will be described first, then each of the more common modes will be described in more detail and examples of typical applications will be utilized to show the particular advantages of the various modes.

Each scanned probe technique relies on a very sharp probe positioned within a few nanometers above the surface of interest. Some combination of probe and/or substrate positioning is required to provide sub-nm-resolution, three-dimensional motion of the probe relative to the substrate. When the probe translates laterally (horizontally) relative to the sample, any change in the height of the surface causes the detected probe signal to change. In general, if the probe signal decreases, this means that the point on the surfacedirectly beneath the probe is farther from the probe than the previous point was. Conversely, if the signal increases, then the point on the surface is closer to the probe than the previous point. The electronic circuit that controls the vertical position of the probe relative to the sample uses these changes in the probe signal as sensory feedback to decide which direction (up or down) to move the probe to maintain a constant probe signal. When the probe signal decreases, the circuit realizes that the surface is now farther away, so it moves the probe down until the signal increases to the same level that was measured at the previous point. Similarly, the circuit responds to increases in probe signal by moving the probe up, away from the surface, until the signal decreases back to the desired level. The distance that the probe is moved up or down to return the probe signal to the desired value is therefore related to the height at each point.

The tutorial will include a discussion of the role of SPM in a microscopy and microanalytical laboratory addressing the commonly asked question: "Do I need AFM if I already have SEM and/or TEM?" 


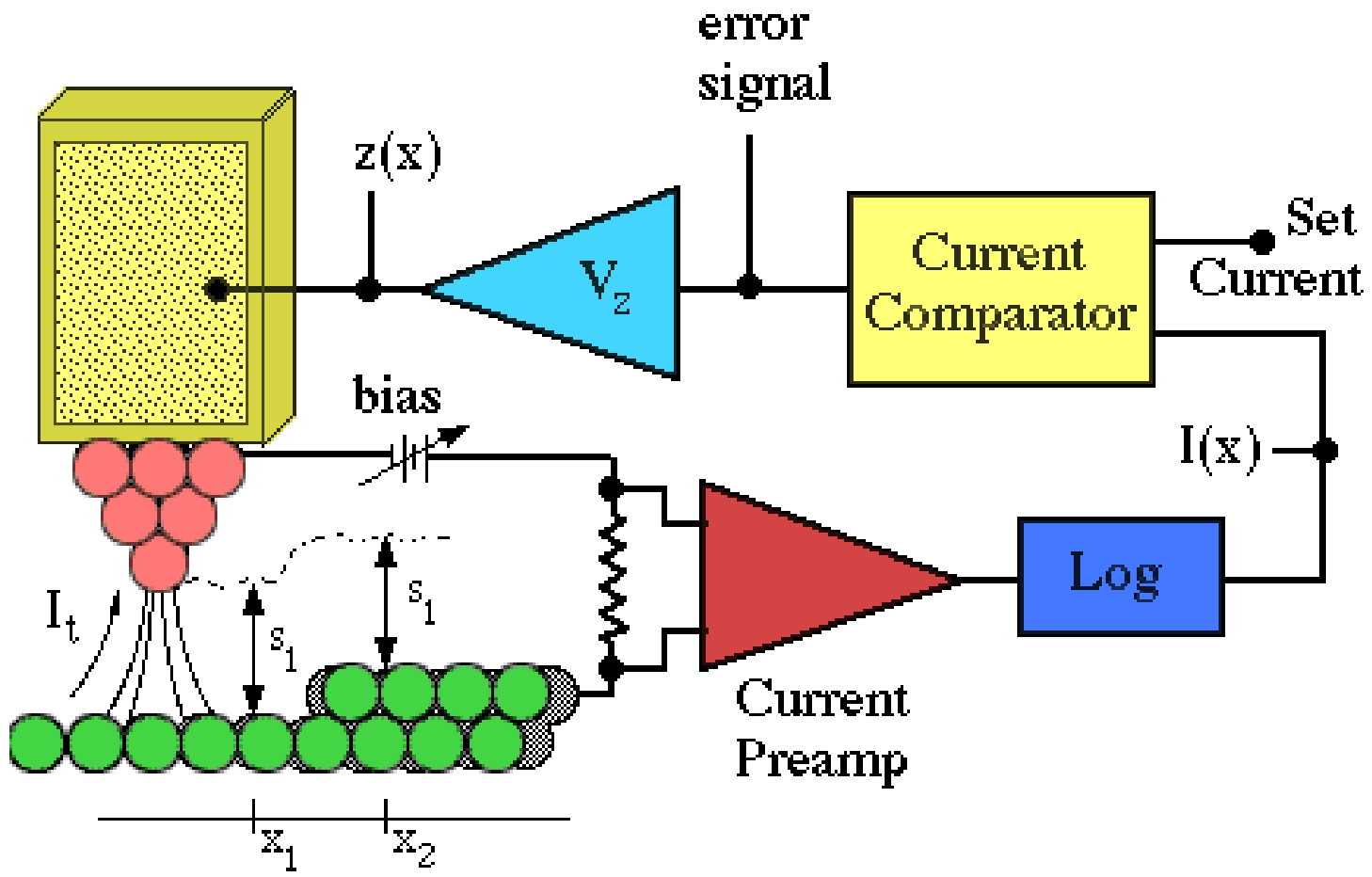

Fig.1. Basic STM concept.

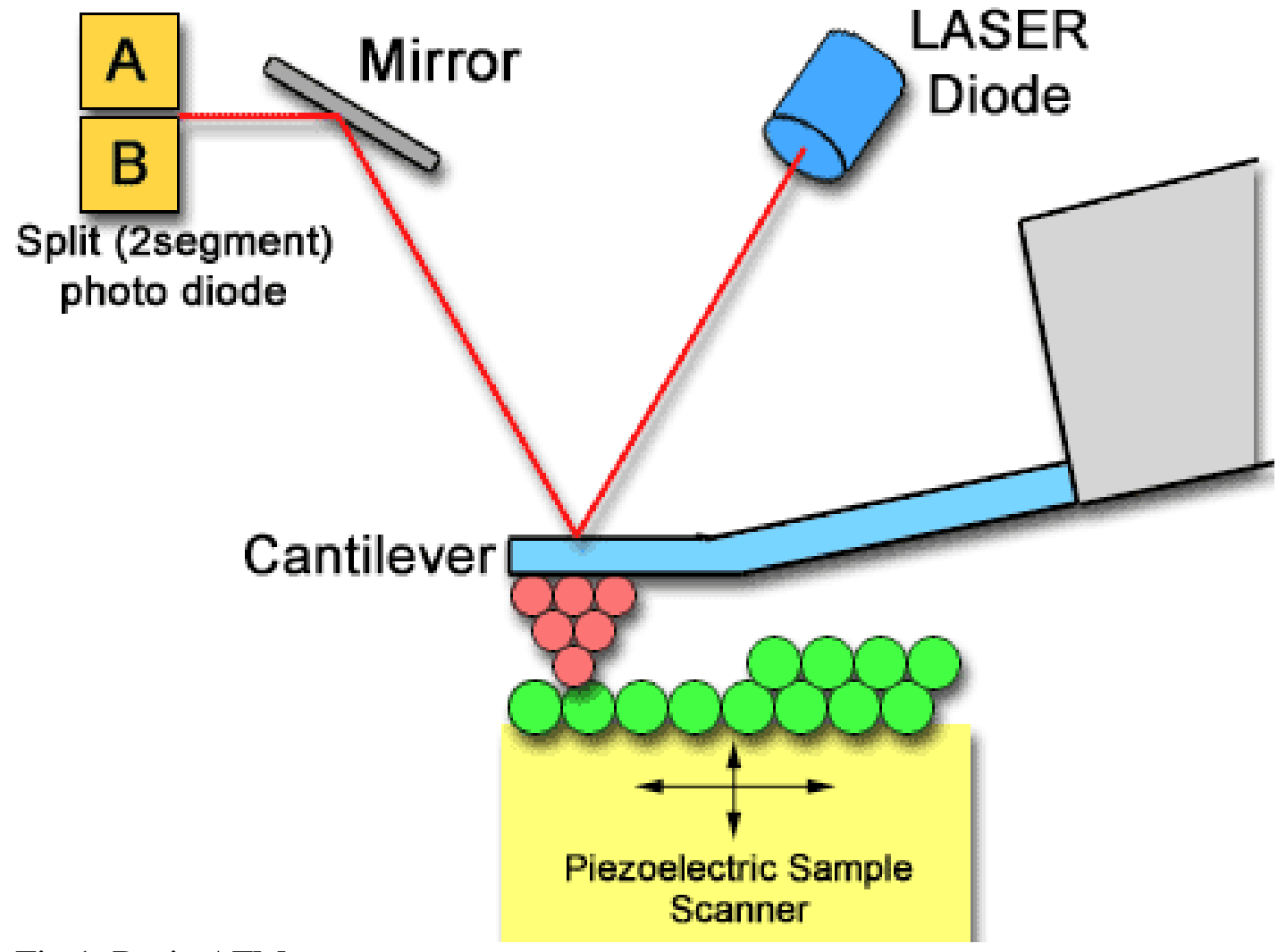

Fig.1. Basic AFM concept. 